\title{
Growing pains
}

\author{
Exoplanetary science is one of the most rapidly developing fields in astronomy and has great near- and medium- \\ term prospects, but various challenges can hinder its growth. The community needs to be prepared to discuss them \\ constructively and openly without spiralling into infighting.
}

Y ear after year, important milestones and exciting discoveries keep exoplanetary science at the frontier of astronomy, popular with the public and prominent in the news. And 2018 is no exception, having already experienced a succession of technological and scientific landmarks.

First of all, this year has been especially rewarding for space-based missions dedicated to exoplanetary science. NASA's next-generation space telescope for exoplanetary detection, the Transiting Exoplanet Survey Satellite (TESS), was launched in April and has just started acquiring science data (see the News \& Views article in this issue). TESS, which is expected to discover approximately 10,000 planets during the two years of its primary mission and has fuel for $\sim 20$ years of operations, will be complemented by ESA's CHaracterising ExOPlanets Satellite (CHEOPS) in the first half of 2019. In addition, the Atmospheric Remote-sensing Infrared Exoplanet Largesurvey (ARIEL) space observatory was selected as ESA's fourth medium-class mission (M4) in March. This choice is all the more impressive as ESA's M3 selection was also a mission with a strong exoplanetary focus (PLAnetary Transits and Oscillations of stars - PLATO). Space agencies usually distribute their few openings for space missions evenly across the various fields of astronomy, so ESA's decision underscores the current momentum of exoplanetary science.

This momentum is also highlighted by the number of ground-based instruments and telescopes that are expected to present their first results this year. Among these are SPECULOOS (Search for habitable Planets EClipsing ULtra-cOOl Stars), which will look for planets around the very promising class of cold stars and brown dwarfs in the solar neighbourhood, and the high spectral resolution spectrometers ESPRESSO (Echelle SPectrograph for Rocky Exoplanet and Stable Spectroscopic Observations), at the Very Large Telescope, and EXPRES (EXtreme PREcision Spectrometer), based at the Discovery Channel Telescope, which will be powerful tools to characterize exoplanetary atmospheres.

Scientific achievements are on a par with this impressive parade of technical advancements. We are now able to observe planets at every stage of their history, as demonstrated by the recent imaging of a planet caught during formation (described in a News \& Views article in this issue). The sample of detected exoplanets and multiplanetary systems (around 3,800 and 630 , respectively) is now big enough to allow researchers to start investigating general trends in planetary populations and systems architectures, in order to determine the nature of the broad physical mechanisms regulating planetary formation and evolution. New categories of exoplanets are being defined, such as the 'ultrahot Jupiters' like KELT-9b (whose extended hydrogen envelope is the subject of the Letter by Fei Yan and Thomas Henning published in this issue). And there is increasing interest in using laboratory measurements to help characterize, among other things, exoplanetary atmospheres and interiors (see, for example, the Letters by Hörst et al. and Smith et al., respectively).

A direct result of this continuous flux of ground-breaking results and missiondriven funding is the rapid growth of the exoplanetary community, not only in numbers but also in scope. New expertise is brought in by researchers coming to work on exoplanetary science, from stellar physicists to planetary scientists, including geologists and geochemists. The increasing need to define the complex concept of habitability more accurately and to develop more rigorous ways to detect - and possibly characterize extraterrestrial life is also pushing for a more inclusive participation of life scientists.

This significant influx of new people at a quick pace generates a series of complications that the community must face. Different fields bring different procedures and different languages: finding a common nomenclature is already a fundamental but by no means simple challenge, as discussed in our Editorial published in February last year. In general, the various groups within the exoplanetary community have to learn how to communicate constructively in order to create productive collaborations. Luckily, there are indications that researchers are aware of this issue and working on it. One approach is to organize specific events to bring these groups together. For example, the Diversis Mundi workshop at the European Southern Observatory in March 2018 was specially conceived to bring the exoplanetary and Solar
System communities together, with a series of keynote 'bridge' talks tackling various topics from both points of view. The need for a more inclusive exoplanetary meeting was also what led to the creation of the Exoplanets Conference Series, as described by Kevin Heng, one of the organizers, in the Nature Research Astronomy Community. Future efforts should be focused on bringing the physical and life scientists together, as such meetings are still rare.

There are, in addition, several more technical issues that are shaping up to be main topics of discussion, and possibly points of contention, in the near future. The widening use of machine learning-related techniques for data analysis in exoplanetary science, of which the Letter by MárquezNeila et al. in this issue is a good example, is currently getting significant attention. In fact, machine learning is undoubtedly powerful, but it can be risky if used blindly as a black box. It is important that an agnostic approach to the method itself is maintained and any end-products, such as transit detections or physical scenarios from atmospheric models, undergo close scrutiny. There is also increasing attention and work devoted to recognizing and properly treating false positives, due for instance to spurious contributions in the transits or to stellar activity. More physics-related issues include the reliability of atmospheric metallicity measurements and our actual knowledge of the equations of state at core conditions, both very important if we want to extract robust conclusions on exoplanetary characterization. The community seems to be reacting to these issues and intense debates are developing around such topics.

In order to cope with all of these challenges and for exoplanetary science to grow into a mature field, the community needs to avoid infighting through frank and constructive discussions and to pursue inclusiveness at all levels. Time will tell if such a collaborative attitude will persist as the community grows - and the competition for finite resources increases. For now, the signs are positive.

Published online: 3 September 2018 https://doi.org/10.1038/s41550-018-0581-2 